\title{
Efficacy of stem cells on bone consolidation of distraction osteogenesis in animal models: a systematic review
}

\section{Carlos Manuel Rubio MORILLO(a) Mariane Cristina SLONIAK(a) Fernanda GONÇALVES ${ }^{(b)}$ Cristina Cunha VILLAR ${ }^{(a)}$}

(a) Universidade de São Paulo - USP, School of Dentistry, Department of Stomatology, São Paulo, SP, Brazil.

(b) Universidade de São Paulo - USP, School of Dentistry, São Paulo, SP, Brazil.

Declaration of Interests: The authors certify that they have no commercial or associative interest that represents a conflict of interest in connection with the manuscript.

Corresponding Author:

Carlos Manuel Rubio Morillo

E-mail:crubio@usp.br

Submitted: April 11, 2018

Accepted for publication: June 27, 2018

Last revision: July 10, 2018
Abstract: Distraction osteogenesis (DO) relies on the recruitment and proliferation of mesenchymal stem cells (MSC) to the target site, where they differentiate into osteoblasts to promote bone formation. Nevertheless, MSC recruitment appears to be slow and limits bone formation in DO defects. Thus, this systematic review aims to evaluate the ability of locally applied MSC to enhance bone formation in DO preclinical models. Databases were searched for quantitative pre-clinical controlled studies that evaluated the effect of local administration of MSC on DO bone formation. Eligible studies were identified and data regarding study characteristics, outcome measures and quality were extracted. Nine studies met the inclusion criteria. Autogenous and xenogenous MSC were used to promote DO bone formation. These included bone marrow-derived MSC, adipose tissue-derived MSC and MSC derived from human exfoliated deciduous teeth. Meta-analysis was not possible due to heterogeneities in study designs. Local MSC implantation was not associated with adverse effects. In 4 out of the 5 studies, locally delivered undifferentiated bone-marrow MSC had a positive effect on DO bone formation. Few studies evaluated the therapeutic effects of MSC from other sources. The adjunct use of biologically active molecules or forced expression of key genes involved in osteogenesis further boosted the ability of bone-marrow MSC to promote DO bone formation. While risk of bias and heterogeneity limited the strength of this systematic review, our results suggest that the use of MSC is safe and may provide beneficial effects on DO bone formation.

Keywords: Mesenchymal Stromal Cells; Osteogeneses, Distraction; Osteogenesis; Review.

\section{Introduction}

Distraction osteogenesis (DO) is a method that induces osseous neoformation between two bone segments surgically separated in response to the application of graduated and controlled traction force throughout the bony gap. ${ }^{1,2}$ This technique have been used in treatment of congenital and acquired craniofacial deformations, as it provides some advantages over traditional autogenic bone grafts, including no need for a second surgical site, reduced operating time and post-operative 
morbidity. ${ }^{2}$ Craniofacial DO outcome depends on multiple factors, including patient's age, the surgical technique (corticotomy or osteotomy), distraction rate and rhythm, latency period, contention period, and the type of the distraction device used (i.e. intraoral, subcutaneous or extraoral) ${ }^{1,2,3,4}$

DO relies on the recruitment and proliferation of mesenchymal stem cells (MSC) to the target site, where they differentiate into osteoblasts to promote bone formation/mineralization. ${ }^{5} \mathrm{MSC}$ recruitment into DO defects is stimulated endogenously by the fracture healing process and exogenously by mechanical distraction. ${ }^{6}$ Nevertheless, under standard circumstances, MSC recruitment appears to be slow and limits the amount of DO bone formation. ${ }^{7}$ Moreover, MSC migration may be further compromised in elderly and under conditions such as poor vascularity, severe trauma and radiotherapy. Along with these lines, DO animal models were developed to evaluate the effect of locally applied MSC on DO bone formation. ${ }^{8,9,10,11,12,13,14,15,16}$ Thus, this systematic review aims to evaluate the following PICO question: "In animals submitted to DO (Participant), how does local MSC administration (Intervention), compared to no MSC administration (Comparison), influence bone consolidation (Outcome)?"

\section{Methodology}

\section{Focused question}

We conducted a systematic review of the literature to address the following PICO question: "In animals submitted to DO (Participant), how does local MSC administration (Intervention), compared to no MSC administration (Comparison), influence bone consolidation (Outcome)?" This systematic review was conducted according to the Preferred Reporting Items for Systematic Reviews and Meta-Analyses (PRISMA) guidelines. ${ }^{17}$

\section{Eligibility criteria}

\section{Type of studies}

Only pre-clinical controlled animal model studies using MSC locally in association with DO were eligible.

\section{Study population}

The population of interest included animals that underwent DO.

Type of intervention and comparison

DO sites treated with MSC were compared to control DO sites that did not receive cells. The protocol of control groups varied according to the type of intervention in each study.

\section{Outcome measures}

The primary outcome was the amount of new bone formation, measured histologically, radiographically or by micro-computed tomography (micro-CT) in DO sites.

\section{Search strategy}

Search strategies were developed for MEDLINE, EMBASE, LILACS, SCIELO, SCOPUS, WEB OF SCIENCE databases, as well as the grey literature. Medical subject headings (MesH) terms were combined with Boolean operators and used to search databases. All searches were performed up to August 2017. The following MeSH terms were used: (((("stem cells" OR "stem cell" OR "progenitor cells" OR "progenitor cell" OR "induced pluripotent stem cells" OR "IPS cells" OR "IPS cell" OR "adult stem cells" OR "pluripotent stem cells" OR "pluripotent stem cell" OR "multipotent stem cells" OR "multipotent stem cell" OR "totipotent stem cells" OR "totipotent stem cell" OR "hematopoietic stem cells" OR "hematopoietic stem cell" OR "mesenchymal stromal cells" OR "mesenchymal stromal cell" OR "mesenchymal stem cells" OR "mesenchymal stem cell" OR "mesenchymal progenitor cells" OR "mesenchymal progenitor cell" OR "bone marrow stromal cells" OR "bone marrow stromal cell" OR "stromal cells" OR "stromal cell" OR "bone marrow cells" OR "bone marrow cell" OR "epithelial mesenchymal transition" OR "cell transplantation" OR "stem cell transplantation" OR "mesenchymal stem cell transplantation" OR "hematopoietic stem cell transplantation" OR "peripheral blood stem cell transplantation")) AND "bone regeneration") AND ("distraction osteogenesis" OR "osteogenic distraction ")) AND (oral OR intraoral), NOT review. Manual searches of reference lists from 
selected full articles complemented the electronic search. Potentially eligible studies were limited to articles published in English, Spanish, and Portuguese.

\section{Exclusion criteria}

Reviews, in vitro, human studies, animal studies without controls were excluded.

\section{Screening methods and data extraction}

Two calibrated reviewers (CMR and FG) screened independently the titles and abstracts. Studies appearing to meet the inclusion criteria, or those with insufficient information in the title and abstract to make a clear decision, were selected for evaluation of the full manuscript, which was done independently by the same reviewers to determine study eligibility. Disagreements were solved by discussion and agreement. Reasons for rejecting studies were recorded for each study. Agreement between reviewers was described by kappa coefficient. The following data were extracted independently by the same reviewers and recorded: citation, year of the publication, MSC origin, characterization, state of differentiation at application, time of transplantation, number of cells transplanted, animal model, number of animals, number of defects, defect type, size, location, scaffold experimental groups, length of follow-up, healing period and results.

\section{Quality assessment}

Quality assessment of included studies was performed independently by two reviewers (CMR and FG), blinded to the name of the authors. Studies were categorized according to the SYRCLE's risk of bias tool for animal studies. ${ }^{18}$

\section{Results}

\section{Study selection}

The computerized search strategy yielded 29 citations, of which 14 were screened for potentially meeting the inclusion criteria $(\mathrm{k}=0.791$; Figure 1$)$. Independent screening of abstracts led to the rejection of 4 articles ( $\mathrm{k}=0.837$; Figure 1$)$. Full texts of the remaining 10 publications were reviewed for possible inclusion. Of these, one article ${ }^{19}$ was further excluded for reasons indicated in Table $1(\mathrm{k}=1.00)$ reference. A manual search of reference lists of selected studies yielded no additional studies (Figure 1). Characteristics of the final nine retained studies are reported in Tables 2 and 3.

\section{Study characteristics}

\section{Experimental animals}

Most studies (6 of 9) used New Zealand white rabbits, ${ }^{8,910,11,12,14}$ one study use Japanese white rabbits. ${ }^{15}$ Finally, two use Sprague-Dawley rats. ${ }^{13,16}$



Figure 1. Simplified search strategy outline.

Table 1. Excluded study.

\begin{tabular}{lc}
\hline Study & Reason for exclusion \\
\hline Zeng et al. $(2016)^{19}$ & Control group did not receive the scaffold used in MSC-based therapy. \\
\hline
\end{tabular}


Table 2. Description of the models used in the included studies.

$\begin{array}{llc}\text { Study } & \text { Animal model } & \begin{array}{c}\text { Cell origin }(n) / \text { characterization/ number of } \\ \text { transplanted cells/ state of cell differentiation } \\ \text { at application/ time of transplantation }\end{array} \quad \text { Defect description/ location/ size }\end{array}$

at application/ time of transplantation

BM-MSC, donor origin was not stated ( $n$ : not informed); ability to adhere; positive for CD29 and CD44 and negative or low

24 New Zealand

Ma et al. white rabbits aged 3

$(2017)^{(8)} \quad$ months and weighing

$2.5-3.0 \mathrm{~kg}$.

$54 \mathrm{OVX}+18$ sham operated female New

Sun et al. Zealand white rabbits, months and weighing approximately $2 \mathrm{~kg}$.

22 skeletally immature New

Alkaisi et al. Zealand white $(2013)^{(10)} \quad$ rabbits aged 3 to 5 months and weighing

12 New Zealand Zhang et al. white rabbits aged $(2012)^{(11)} \quad$ approximately 4 months. expression of CD34 and CD45; ability to undergo osteogenic and adipogenic differentiation; aifferentiated into an osteogenic lineage at application; $5 \times 10^{6}$ cells/defect; injected into the distraction gap at the end of the DO period.

Autogenous ADSC ( $n=36$ ); fibroblast-like cell morphology; $1 \times 10^{7}$ cells/defect; undifferentiated at application; injected into the distraction gap at the end of the DO period.

Xenogenous SHED $(n=4)$; fibroblast-like cell morphology; ability to adhere; colony forming efficacy; positive for CD105 and

CD 166; $6 \times 10^{6}$ cells/defect; undifferentiated at application; cells were transplanted at the surgical appointment.

BM-MSC, donor origin was not stated $(n=$ not informed); ability to undergo osteogenic, adipogenic, and chondrogenic differentiation; ability to adhere and colony forming efficiency; number of implanted cells was not informed; undifferentiated at application; applied into the distraction gap at the end of the DO period.

54 skeletally mature male New Zealand

Lai et al. white rabbits weighing $3.0-3.5 \mathrm{~kg}$.

42 skeletally mature Jiang et al. male New Zealand $(2010)^{(14)}$ white rabbits weighing

$$
2.0-3.0 \mathrm{~kg}
$$

12 adult male Japanese white

Kinoshita et abbits weighing 3.0 $-3.4 \mathrm{~kg}$.

54 adult male Hu et al. Sprague-Dawley $(2007)^{(13)} \quad$ rats weighing rats weighing $350-400 \mathrm{~g}$

Qi et al.

40 adult male $(2006)^{(16)}$ weighing 350-400 g. $(2014)^{(9)} \quad$ aged approximately 3 approximately $2.7 \mathrm{~kg}$ Sprague-Dawley rats $1 \times 10^{7}$ cells/defect; undifferentiated at application; applied into the distraction gap at the end of the DO period.

Autogenous BM-MSC Ability to adherence;

Autogenous BM-MSC; ability to adhere;

$1 \times 10^{7}$ cells/defect; undifferentiated at application; injected into the distraction gap at the end of the DO period.

ability to undergo osteogenic differentiation

$1 \times 10^{7}$ cells/defect; differentiated into

osteogenic lineage at application; applied into the distraction gap at the end of the DO period.

Autogenous BM-MSC; Fibroblast-like cell morphology; ability to adhere; colony forming efficacy; 1 x106 cells/defect; undifferentiated at application; applied into the distraction gap at the end of the DO period.

Autogenous BM-MSC; fibroblast-like cell morphology, ability to adhere, colony forming efficacy; $5 \times 10^{5}$ cells/defect undifferentiated at application; applied into the distraction gap at the end of the DO period.
An osteotomy line was made between the first premolar and the mental foramen of the left mandible. An external distractor was fixed across both cortices. Left jaw underwent osteotomy by a fissured bur. After three days, the distractor was activated at a rate of $10 \mathrm{~mm} /$ twice a day, for five days, resulting in a $10 \mathrm{~mm}$ distraction gap

The corticotomy was performed immediately anterior to the first premolar. The distractor was fixed and distraction started after seven days, with an activation rate of $0.8 \mathrm{~mm} /$ day for ten days, resulting in $8 \mathrm{~mm}$ distraction gap.

A longitudinal incision was made on the inferior border of the right mandible. An incomplete osteotomy cut was placed between the first premolar and the mental foramen. A $12 \times 12 \mathrm{~mm}$ distraction device was fixed to either side of the osteotomy cut.After four days, the distraction device was activated at a rate of $1.0 \mathrm{~mm} /$ day for six days, resulting in a $6 \mathrm{~mm}$ distraction gap.

A straight unilateral osteotomy was performed from the first premolar to the mental foramen. A custom-made distractor was placed and fixed perpendicularly to the plane of the osteotomy. After one week, the distraction device was activated at a rate of $0.9 \mathrm{~mm} /$ day for 11 days, resulting in a $10 \mathrm{~mm}$ distraction gap.

The osteotomy was performed on the bucal aspect of the left anterior mandible, anterior to the first molar. A custom-made distractor was fixed with 4 self-tapping activated at a rate of $0.4 \mathrm{~mm} / 12$ hours for 6 days, resulting in a $4.8 \mathrm{~mm}$ distraction gap.

An osteotomy line was made on the right mandibles, between the first premolar and the mental foramen.

A custom-made titanium external distractor was fixed across both cortices. After three days, the distraction device was activated at a rate of $2.0 \mathrm{~mm} /$ day for 5 days, resulting in a $10 \mathrm{~mm}$ distraction gap.

Osteotomies were made bilaterally on the zygomatic process of the maxilla, and custommade distractor devices were fixed. After five days, the distraction device was activated at a rate of $2.0 \mathrm{~mm} /$ day for 4 days, resulting in an $8 \mathrm{~mm}$ distraction gap.

The osteotomy was performed to the middle of the anterior ramus to the inferior border of the right mandible; After five days, the distraction device was activated at a rate of $0.4 \mathrm{~mm} /$ day for 8 days, resulting in a $3.2 \mathrm{~mm}$ distraction gap.

Osteotomy was performed from the upper border of the anterior ramus to the inferior border of the mandible. A custom made distractor was fixed to osteotomy site. After five days, the distraction device was activated at a rate of $0.4 \mathrm{~mm} /$ day, for 8 days, resulting in a $3.2 \mathrm{~mm}$ distraction gap. screws. After six days, the distraction device was

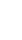


Table 3. Summarized outcomes of the included studies.

\begin{tabular}{|c|c|c|c|}
\hline Study & Groups/ $n$ of defects & Healing period & Outcomes \\
\hline $\begin{array}{l}\text { Ma et al. } \\
(2017)^{(8)}\end{array}$ & $\begin{array}{l}\text { Group 1: } B M-M S C+H A(n=8) \\
\text { Group 2: } B M-M S C-C S F+H A(n=8) \\
\text { Group 3: oBM-MSC-CSF }+H A(n=8)\end{array}$ & 6 weeks & $\begin{array}{l}\text { Micro-CT } \\
\text { New bone }\left(\mathrm{mm}^{3}\right): \\
\text { BM-MSC + HA: } \sim 360 \text { (A) } \\
\text { BM-MSC-CSF + HA: } 445 \pm 29.6 \text { (B) } \\
\text { oBM-MSC-CSF + HA: } 510 \pm 26.3 \text { (C) } \\
\text { oBM-MSC group exhibited a more mature cortical bone. }\end{array}$ \\
\hline $\begin{array}{l}\text { Sun et al. } \\
(2014)^{(9)}\end{array}$ & $\begin{array}{l}\text { Group 1: OVX rabbits + saline solution } \\
\text { ( } n=6 / \text { time point). } \\
\text { Group 2: OVX rabbits + Adv-GFP- } \\
\text { ADSC ( } n=6 \text { /time point). } \\
\text { Group 3: OVX rabbits + Adv-Runx2- } \\
\text { GFP-ADSC ( } n=6 / \text { time point). } \\
\text { Group 4: sham rabbits }+ \text { saline } \\
\text { solution ( } n=6 / \text { time point). }\end{array}$ & $\begin{array}{c}3,6 \text {, and } 9 \\
\text { weeks }\end{array}$ & 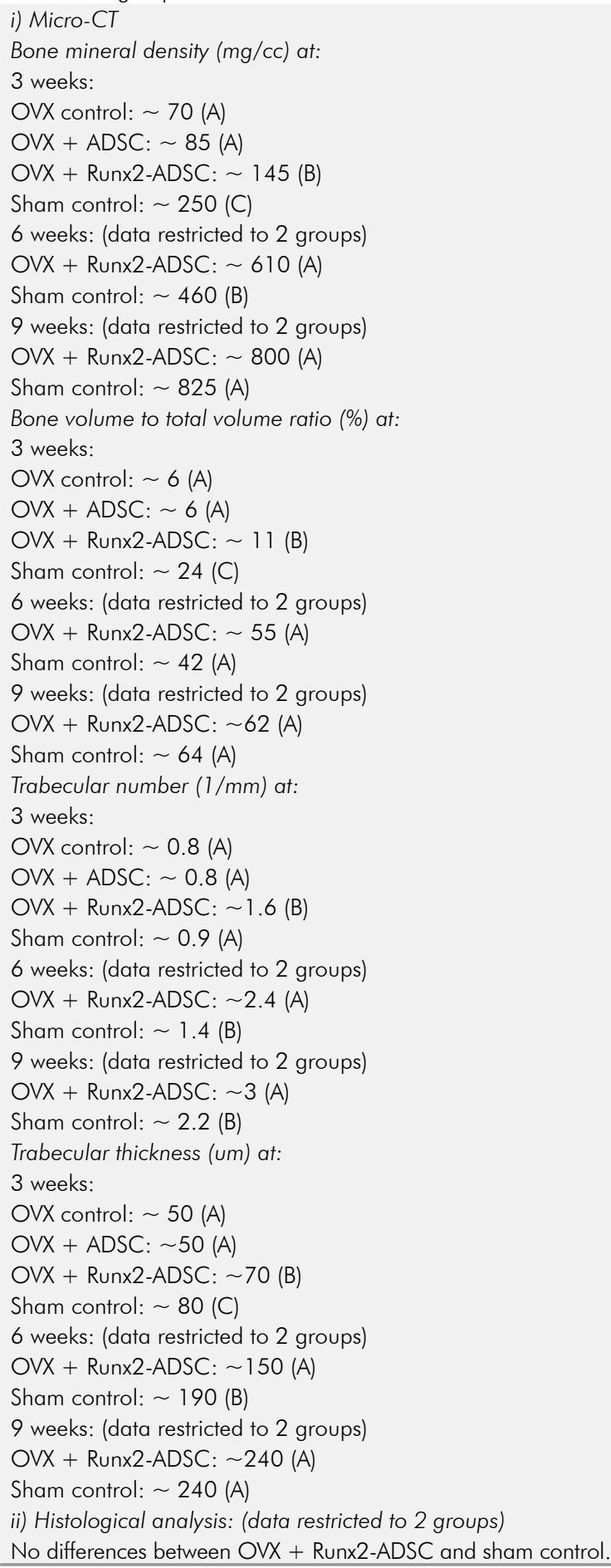 \\
\hline
\end{tabular}


Alkaisi et al. Group 1: negative control $(n=9)$. $(2013)^{(10)} \quad$ Group 2: SHED $(n=9)$.

2,4 , and 6
weeks

i) Radiographic analysis

2 weeks: SHED group had more bone radiopacity bridging the DO gap.

4 weeks: SHED group demonstrate bony continuity with greater radiodensity than control group.

6 weeks: SHED group demonstrated greater radiodensity in the distraction gap than that the control group.

ii) Histomorphometric analysis.

New Bone (\%) at:

2 weeks:

Control: $18.41(\mathrm{~A})$

SHED: 35.97 (B)

4 weeks:

Control: $31.45(\mathrm{~A})$

SHED: 56.69 (B)

6 weeks:

Control: $52.61(\mathrm{~A})$

SHED: 65.28 (B)

Bone union at:

2 weeks:

Control: 0.96 (A)

SHED: 1.75 (B)

4 weeks:

Control: $1.58(\mathrm{~A})$

SHED: 2.96 (B)

6 weeks:

Control: 2.5 (A)

SHED: 3.25 (B)

Stage of bone maturity at:

2 weeks:

Control: 2.25 (A)

SHED: 3.16 (B)

4 weeks:

Control: 3.25 (A)

SHED: 4.5 (B)

6 weeks:

Control: 3.5 (A)

SHED: 4.66 (B)

i) Radiographic analysis

RT + MSC and RT + MSC BMP-2/7 BM-MSC groups showed more cortical bone formation compared with sham-RT and RT control groups.

ii) Micro-CT

Trabecular separation $(\mathrm{mm})$ :

Sham-RT: $0.99 \pm 0.16(\mathrm{~A})$

RT: $1.00 \pm 0.07(A)$

RT + BM-MSC: $0.99 \pm 0.17$ (A)

RT + BMP2/7 BM-MSC: $1.32 \pm 0.17$ (B)

Trabecular thickness $(\mathrm{mm})$ :

Sham-RT: $0.12 \pm 0.01$ (A)

RT: $0.12 \pm 0.03(A)$

RT + BM-MSC: $0.15 \pm 0.01$ (A)

RT + BMP2/7_BM-MSC: $0.22 \pm 0.02$ (A)

Number of trabeculae $(1 / \mathrm{mm})$ :

Sham-RT: $0.91 \pm 0.12(\mathrm{~A})$

RT: $0.90 \pm 0.04$ (A)

RT + BM-MSC: $0.83 \pm 0.04$ (A)

RT + BMP2/7 BM-MSC: $1.08 \pm 0.1$ (A)

Bone volume fraction:

Sham-RT: $0.12 \pm 0.03(\mathrm{~A})$

RT: $0.11 \pm 0.03(\mathrm{~A})$

RT + BM-MSC: $0.08 \pm 0.02(\mathrm{~A})$

RT + BMP2/7 BM-MSC: $0.12 \pm 0.04$ (A)

iii) Histological analysis

BM-MSC and BMP2/7_BM-MSC exhibited more mature medullary and cortical bones than the control groups. 
Continuação

Lai et al.

$(2011)^{(12)}$

Jiang et al. $(2010)^{(14)}$

Group 1: Saline solution $(n=14)$.

Group 2: Ad5 BM-MSC $(n=14)$.

Group 3: Ad5_bFGF BM-MSC $(n=14)$.
2 and 6 weeks point).

Group 2: BM-MSC ( $n=9$ /time point). Group 3: OSX_BM-MSC ( $n=9$ /time point). i) Radiographic analysis

Radiodensity at:

2 weeks:

Control: $\sim 54$ (A)

BM-MSC: $\sim 75$ (B)

OSX BM-MSC: $\sim 95$ (C)

6 weeks:

Control: 105 (A)

BM-MSC: 121 (B)

OSX_BM-MSC: $\sim 143(\mathrm{C})$

ii) Histological analysis.

Trabecular thickness $(\mathrm{mm})$ at:

2 weeks:

Control: $22.89 \pm 1.96(\mathrm{~A})$

BM-MSC: $26.96 \pm 3.23$ (B)

OSX BM-MSC: $39.39 \pm 3.54$ (C)

6 weeks:

Control: $34.69 \pm 3.73$ (A)

BM-MSC: $42.41 \pm 2.34$ (B)

OSX BM-MSC: $54.21 \pm 2.38$ (C)

Newly formed cortical bone area (\%)at:

2 weeks:

Control: $44.53 \pm 5.43$ (A)

BM-MSC: $52.43 \pm 2.74$ (B)

OSX_BM-MSC: $61.62 \pm 3.10$ (C)

6 weeks:

Control: $84.42 \pm 3.06$ (A)

BM-MSC: $90.26 \pm 2.12$ (B)

OSX BM-MSC: $97.32 \pm 2.65$ (C)

Cancellous bone area (\%) at:

2 weeks:

Control: $24.54 \pm 3.22$ (A)

BM-MSC: $33.85 \pm 3.49$ (B)

OSX BM-MSC: $42.45 \pm 2.98$ (C)

6 weeks:

Control: $42.05 \pm 3.58$ (A)

BM-MSC: $53.80 \pm 2.91$ (B)

OSX_BM-MSC: $6 \overline{7} .75 \pm 4.04$ (C)

i) DXA analysis

Bone mineral density $\left(\mathrm{g} / \mathrm{cm}^{2}\right)$ :

Control: $\sim 0.23(\mathrm{~A})$

Ad5 BM-MSC: $\sim 0.25$ (B)

Ad5_bFGF BM-MSC: $\sim 0.27$ (C)

Bone mineral content $(\mathrm{g})$ :

Control: $\sim 0.21$ (A)

Adv5 BM-MSC: $\sim 0.23$ (B)

Ad5 bFGF BM-MSC: $\sim 0.25$ (C)

ii) Micro-CT

Bone volume to total volume ratio (\%):

Control: $56.00 \pm 3.72$ (A)

Adv5 BM-MSC: 61.6 (B)

Ad5 bFGF BM-MSC: 67.2 (C)

Connectivity density $\left(\mathrm{mm}^{3}\right)$ :

Control: $11.05 \pm 1.73(\mathrm{~A})$

Adv5 BM-MSC: 13.26 (B)

Ad5_bFGF BM-MSC: $\sim 21.00$ (C)

Trabecular thickness $(\mathrm{mm})$ :

Control: $0.21 \pm 0.04(\mathrm{~A})$

Adv5 BM-MSC: $\sim 0.30$ (B)

Ad5-bFGF BM-MSC: 0,38 (C)

Trabecular separation $(\mathrm{mm})$ :

Control: $0.32 \pm 0.03(\mathrm{~A})$

Adv5 BM-MSC: $\sim 0.32(\mathrm{AB})$

Ad5_bFGF BM-MSC: 0,36 (B)

Trabecular number:

Control: $2.01 \pm 0.39$ (A)

Adv5 BM-MSC: $\sim 2.60$ (B)

Ad5_bFGF BM-MSC: 3.28 (C)

iii) Histological analysis (data restricted to half of the samples). While only disordered tiny trabeculae were sporadically found in the distraction gaps of group 1, the distraction gaps in group 3 were completely bridged with mature and regular trabecular bone. Bone trabeculae only partially filled distracted gaps in group 2 . 
Continuação

Kinoshita et

al. $(2008)^{(15)}$
Group 1: PRP ( $\mathrm{n}=6$ defects).

Group 2: Saline solution -contralateral internal control for group 1 ( $n=6$

defects).

Group 3: oBM-MSC + PRP $(\mathrm{n}=6$

defects).

Group 4: Saline solution - contralateral internal control for group 3 ( $n=6$ defects).
Hu et al. $(2007)^{(13)}$
Group 1: Saline solution $(\mathrm{n}=9$ /time point).

Group 2: pEGFP-BM-MSC ( $\mathrm{n}=9$ /time point).

Group 3: pEGFP-BMP-2 BM-MSC (n $=9$ /time point).

$\begin{array}{cl}2,3 \text { and } 4 & \text { i) Radiographic analysis } \\ \text { weeks } & \text { Radiodensity (mm Al) at: }\end{array}$

2 weeks:

Ratio PRP/saline control: $\sim 0.8$ (A)

Ratio BM-MSC + PRP/saline control: $\sim 1.07$ (B)

3 weeks:

Ratio PRP/saline control: $\sim 0.7$ (A)

Ratio BM-MSC + PRP/saline control: $\sim 1.09$ (B)

4 weeks:

Ratio PRP/saline control: $\sim 0.6$ (A)

Ratio BM-MSC + PRP/saline control 1.1 (B)

ii) Histological analysis (data restricted to week 4)

New bone (\%):

Ratio PRP/saline control: $\sim 1.2(\mathrm{~A})$

Ratio BM-MSC + PRP/saline control: $\sim 1.6$ (B)

Bony content in the newly formed bone (\%):

Ratio PRP/saline control: $\sim 0.95$ (A)

Ratio BM-MSC + PRP/saline control: 1.2 (B)

2 and 6 weeks i) Radiographic analysis

Radiodensity

2 weeks:

Control: $\sim 52$ (A)

BM-MSC: $\sim 58$ (B)

BMP-7 BM-MSC: $\sim 70(\mathrm{C})$

6 weeks:

Control: $\sim 99$ (A)

BM-MSC: $\sim 113$ (B)

BMP-2 BM-MSC: $\sim 135$ (C)

ii) Histomorphometric analysis

Newly formed cortical bone area (\%) at:

2 weeks

Control: $43.52 \pm 6.11$ (A)

BM-MSC: $51.28 \pm 3.85$ (B)

BMP-2_BM-MSC: $60.59 \pm 4.00$ (C)

6 weeks

Control: $82.66 \pm 2.06(\mathrm{~A})$

BM-MSC: $89.30 \pm 1.95$ (B)

BMP-2: $95.54 \pm 2.07$ (C)

Cancellous bone area (\%) at:

2 weeks

Control: $23.69 \pm 2.60(\mathrm{~A})$

BM-MSC: $32.22 \pm 4.22$ (B)

BMP-2_BM-MSC: $41.54 \pm 3.01$ (C)

6 weeks

Control: $41.46 \pm 4.01$ (A)

BM-MSC: $52.47 \pm 1.93(\mathrm{~B})$

BMP-2 BM-MSC: $66.33 \pm 4.00$ (C)

Trabecular thickness $(\mathrm{mm})$ at:

2 weeks:

Control: $21.13 \pm 1.05(\mathrm{~A})$

BM-MSC: $25.43 \pm 2.10$ (B)

BMP-2 BM-MSC: $37.32 \pm 3.39$ (C)

6 weeks:

Control: $32.45 \pm 4.33$ (A)

BM-MSC: $40.97 \pm 2.52$ (B)

BMP-2 BM-MSC: $52.32 \pm 3.12$ (C) 


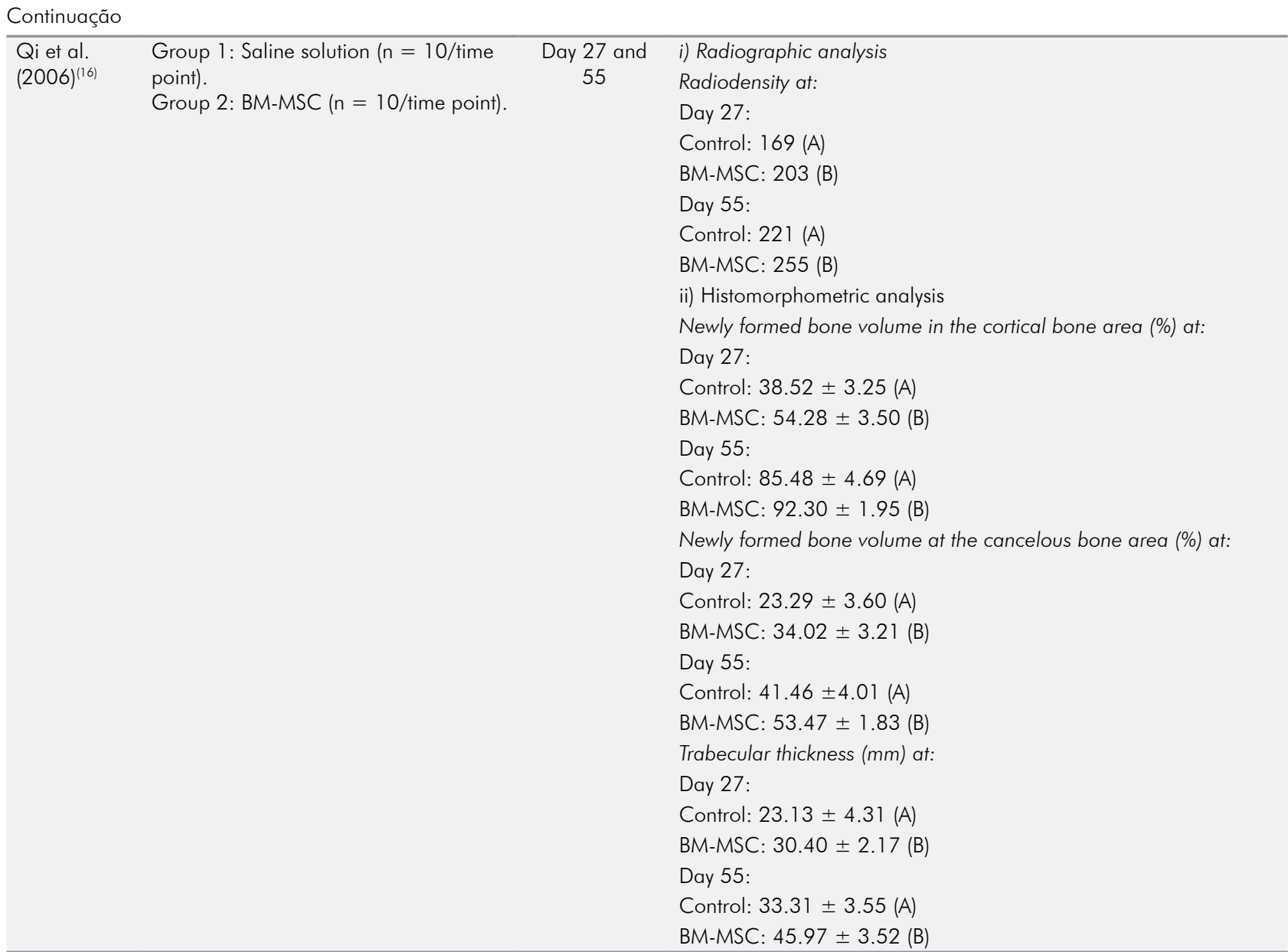

\section{Experimental models}

DO defects were surgically created in all studies. Nonetheless, differences in defect location and morphology were identified among the studies. In five studies, the osteotomy line was made between the first premolar and the mental foramen. ${ }^{8,10,11,12,14} \mathrm{In}$ other two studies, the osteotomy line was made from the upper border of the anterior ramus to the inferior border of the mandible. ${ }^{13,16}$ Finally, in the remaining two studies, the osteotomy cut was made anterior to the first molar ${ }^{9}$ and bilaterally on the zygomatic process of the maxilla. ${ }^{15}$

The activation rate also varied among the studies. More specifically, activation rates of $0.4 \mathrm{~mm} /$ day, $_{1}^{13,16}$ $0.8 \mathrm{~mm} /$ day, $^{9,12} 0.9 \mathrm{~mm} /$ day, $^{11} 1 \mathrm{~mm} /$ day $^{8,10}$ and $2 \mathrm{~mm} /$ day $^{14,15}$ were reported. The resulting DO gap defect measured $10 \mathrm{~mm},{ }^{8,11,14} 8 \mathrm{~mm},{ }^{9,15} 6 \mathrm{~mm}^{10} 4.8 \mathrm{~mm}^{12}$ and $3.2 \mathrm{~mm}^{13,16}$ in the included studies.
Mesenchymal stem cells and scaffolds

Both autogenous $\mathrm{s}^{9,12,13,14,15,16}$ and xenogenous ${ }^{10}$ MSC were used in the studies. Two trials failed to inform the donor origin of the cells. ${ }^{8,11}$ In regard to the source of the cells, bone marrow-derived MSC (BM-MSC) were utilized in seven trials, ${ }^{8,11,12,13,14,15,16}$ while adipocyte-derived MSC (ADSC) ${ }^{9}$ and stem cells derived from human exfoliated deciduous teeth (SHED) ${ }^{10}$ were used in one trial each. In most studies, MSC were characterized by plastic adherent capacity, ${ }^{8,10,11,12,13,14,15,16}$ fibroblastlike morphology, ${ }^{9,10,13,16}$ colony forming ${ }^{10,11,13,16}$ and multipotency $y^{8,11,13,16}$ abilities. In only two studies, MSC were phenotypically characterized using flow cytometry to detect expression of mesenchymal markers. MSC were characterized as positive for $\mathrm{CD} 29,{ }^{8} \mathrm{CD} 44,{ }^{8} \mathrm{CD} 115,{ }^{10} \mathrm{CD} 166,{ }^{10}$ and negative for $\mathrm{CD} 34^{8}$ and $\mathrm{CD} 45 .{ }^{8}$ 
Table 4. Risk of bias in individual studies, assessed using the SYRCLE tool.

\begin{tabular}{|c|c|c|c|c|c|c|c|c|c|c|}
\hline & 1 & 2 & 3 & 4 & 5 & 6 & 7 & 8 & 9 & 10 \\
\hline Study & $\begin{array}{c}\text { Selection } \\
\text { bias } 1\end{array}$ & $\begin{array}{l}\text { Selection } \\
\text { bias } 2\end{array}$ & $\begin{array}{l}\text { Selection } \\
\text { bias } 3\end{array}$ & $\begin{array}{c}\text { Performance } \\
\text { bias } 1\end{array}$ & $\begin{array}{l}\text { Performance } \\
\text { bias } 2\end{array}$ & $\begin{array}{c}\text { Detection } \\
\text { bias } 1\end{array}$ & $\begin{array}{l}\text { Detection } \\
\text { bias } 2\end{array}$ & $\begin{array}{c}\text { Attrition } \\
\text { bias }\end{array}$ & $\begin{array}{l}\text { Reporting } \\
\text { bias }\end{array}$ & $\begin{array}{l}\text { Other } \\
\text { bias }\end{array}$ \\
\hline Ma et al. $(2017)^{8}$ & $?$ & $\checkmark$ & $x$ & $?$ & $x$ & $x$ & $x$ & $\checkmark$ & $\checkmark$ & $\checkmark$ \\
\hline Sun et al. $(2014)^{9}$ & $x$ & $\checkmark$ & $x$ & $?$ & $x$ & $x$ & $x$ & $x$ & $\checkmark$ & $\checkmark$ \\
\hline Alkaisi et al. $(2013)^{10}$ & $?$ & $\checkmark$ & $x$ & $?$ & $x$ & $x$ & $x$ & $x$ & $\checkmark$ & $\checkmark$ \\
\hline Zhang et al. $(2012)^{11}$ & $?$ & $\checkmark$ & $x$ & $?$ & $x$ & $x$ & $\checkmark$ & $x$ & $\checkmark$ & $\checkmark$ \\
\hline Lai et al. $(2011)^{12}$ & $?$ & $\checkmark$ & $\mathrm{x}$ & $?$ & $x$ & $x$ & $x$ & $x$ & $\checkmark$ & $\checkmark$ \\
\hline Jiang et al. $(2010)^{14}$ & $?$ & $\checkmark$ & $x$ & $?$ & $x$ & $x$ & $x$ & $x$ & $\checkmark$ & $\checkmark$ \\
\hline Kinoshita et al. $(2008)^{15}$ & $?$ & $\checkmark$ & $\mathrm{x}$ & $?$ & $x$ & $\mathrm{x}$ & $\mathrm{x}$ & $\mathrm{x}$ & $\checkmark$ & $\checkmark$ \\
\hline Hu et al. $(2007)^{13}$ & $?$ & $\checkmark$ & $x$ & $?$ & $x$ & $x$ & $x$ & $\checkmark$ & $\checkmark$ & $?$ \\
\hline Qi et al. $(2006)^{16}$ & $?$ & $\checkmark$ & $x$ & $?$ & $x$ & $x$ & $x$ & $\checkmark$ & $\checkmark$ & $\checkmark$ \\
\hline
\end{tabular}

IRB, institutional review board; $1 \checkmark=$ Adequate randomization; ? = randomized but no details; $\mathrm{s}=$ no evidence of randomization; $2 \checkmark=$ Baseline characteristics given; $x=$ baseline characteristics not given; $3 \checkmark=$ Evidence of adequate concealment of groups; $x=$ no evidence of adequate concealment of groups; $4 \boldsymbol{V}$ = Evidence of random housing of animals; ? = unknown housing arrangement; $5 \boldsymbol{V}=$ Evidence of examiners blinded to intervention; $x=$ no evidence of examiners blinded to intervention; $6 \checkmark=$ Evidence of random selection for assessment; $\mathrm{x}=$ no evidence of random selection for assessment; $7 \checkmark=$ Evidence of researchers blinded; $\mathrm{x}=$ no evidence of assessor blinded; $8 \checkmark=$ Explanation of missing animal data; $x=$ no explanation of missing animal data; $9 \checkmark=$ Free of selective reporting based on methods/results; $x$ = selective reporting; $10 \checkmark=$ Evidence of random housing of animals; ? = unknown housing arrangement.

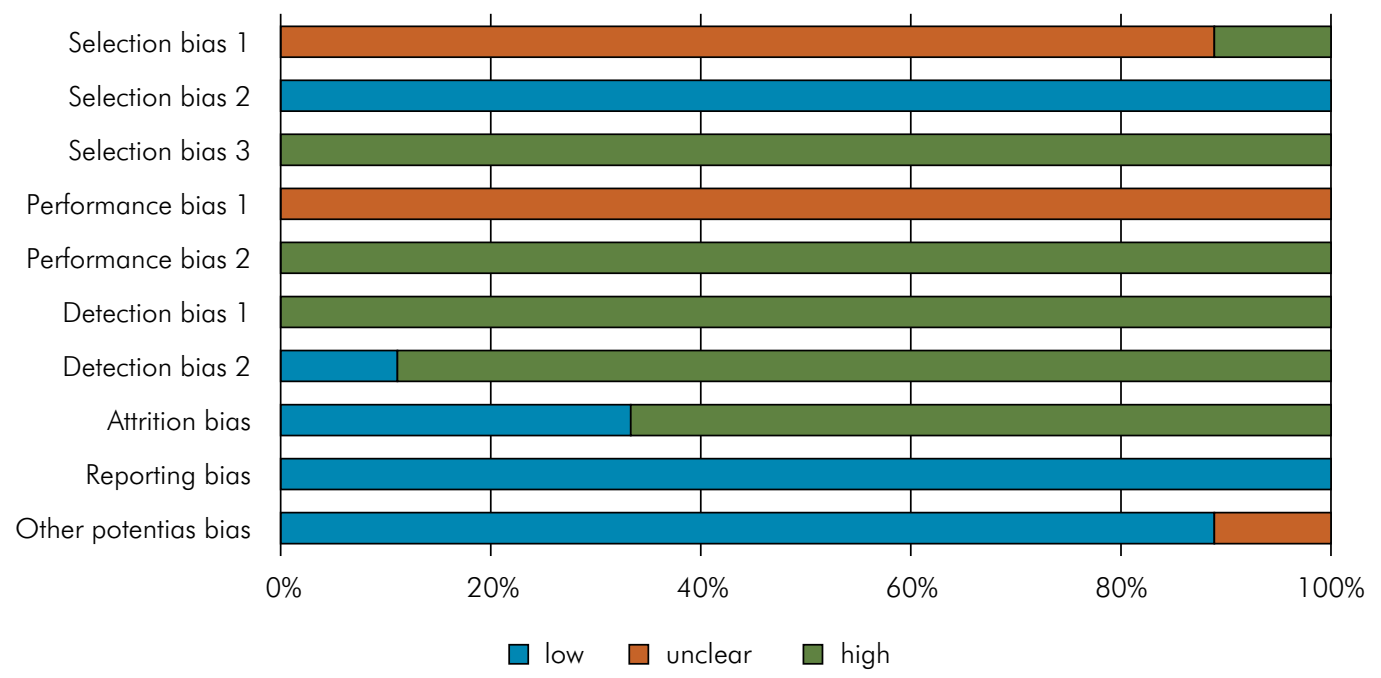

Figure 2. Risk of bias score for each risk item in animal studies, as assessed using the SYRCLE tool.

In all but one study, MSC were locally injected at the end of DO period. ${ }^{9,11,12,13,14,15,16}$ The exception was the study from Alkaisi et al. ${ }^{10}$ in which SHED cells were locally delivered at the surgical appointment. Scaffolds were only employed in two studies. ${ }^{8,15}$

Owing to the high degree of methodological heterogeneity among the included studies no meta-analysis was performed.

\section{Safety}

Overall, the majority of experimental procedures were well tolerated by most animals. Nonetheless, some authors refer to develop of infections, ${ }^{9,13,16}$ mobility of the distraction devices, ${ }^{9,10}$ changes in occlusal relationships ${ }^{10}$ and overgrowth of the incisors. ${ }^{12}$ Most of these reported adverse effects appeared to be 
related exclusively to the $\mathrm{DO}$ procedure. Two studies failed to provide information on adverse effects. ${ }^{8,11}$

\section{Quality Assessment}

The use of the SYRCLE risk of bias tool to assess quality of animal studies indicated a high risk of bias for most studies in the majority of categories (Table 4 and Figure 2). Only two categories, baseline characteristics and reporting bias, were assessed as having a low risk of bias for the majority of studies.

\section{Effect of mesenchymal stem cells on DO bone formation}

\section{Effect of undifferentiated mesenchymal stem cells on bone formation following DO}

Most of the studies included in this systematic review report the effect of undifferentiated MSC on DO bone formation., ${ }^{910,11,12,13,14,16}$ More specifically, five studies evaluated the effect of undifferentiated BM-MSC on DO bone formation, one study used undifferentiated ADSM and another used undifferentiated SHED cells.

Out of the five studies that evaluated undifferentiated BM-MSC, four showed a positive effect of these cells on DO bone formation. ${ }^{12,13,14,16}$ The positive effect of undifferentiated BM-MSC on DO bone formation was demonstrated by radiographic determination of cortical bone formation, ${ }^{11}$ bone mineral content ${ }^{14}$ and bone density. ${ }^{12,14,16}$ Moreover, histological demonstration of increased bone formation by undifferentiated BM-MSC was shown by increased a. cortical bone formation;, ${ }^{12,3,16}$ b. cancellous bone formation; ${ }^{12,13,16}$ and c. trabecular thickness. ${ }^{12,13,16}$ Corroborating these results, micro-CT analysis also showed that the use of undifferentiated BM-MSC was associated with increased new bone, bone volume ratio, connectivity density, trabecular thickness and trabecular number ${ }^{14}$ in DO defects. In sharp contrast, in one study, BM-MSC failed to promote increased bone formation in DO gaps, as compared to the control treatment. ${ }^{11}$

Only one study evaluated the effect of undifferentiated SHED cells on new bone formation in DO gap defects. Histological and radiographic analysis demonstrated that the use of these cells resulted in increased bone formation and bone density, respectively. ${ }^{10}$ Finally, a micro-CT study failed to demonstrated a positive effect of undifferentiated ADSC on DO bone formation, as compared to a control group. ${ }^{9}$

\section{Effect of pre-differentiated MSC on bone formation following DO}

Only one study reported on the use of osteogenically differentiated BM-MSC for DO bone formation. In this study, micro-CT analysis demonstrated that osteogenically pre-differentiated BM-MSC arranged in cell sheets promoted greater bone formation than undifferentiated BM-MSC sheets and negative control treatment. ${ }^{8}$ Moreover, according to histological evaluations, the use of osteogenically differentiated BM-MSC cell sheets induced the formation of a more mature cortical bone. ${ }^{8}$

The combined effect of undifferentiated MSC and biologically active factors

Biologically active factors used in association with undifferentiated MSC included: transcription factors Runt-related transcription factor 2 (Runx 2$)^{9}$ and Osterix (OSX), ${ }^{12}$ bone morphogenetic protein $(\mathrm{BMP})^{11,13}$ and basic fibroblast growth factor (bFGF) ${ }^{14}$

A micro-CT study demonstrated that Runx2 transfected ADSC promoted increased bone mineral density, bone volume, trabecular number and trabecular thickness in DO defects, as compared to control treatment with no adjunct use of cells. ${ }^{9}$ Moreover, the same study showed that forced expression of Runx2 increased the ability of ADSC to promote bone formation. ${ }^{9}$ Likewise, OSX transfected BM-MSC promoted increased cortical and cancellous bone formation, trabecular thickness and radiographic bone density in DO defects, as compared to control treatment. ${ }^{12}$ Forced OSX expression also increased the ability of BM-MSC to promote bone formation in $\mathrm{DO}$ defects. ${ }^{12}$

Conflicting results were reported on the use of BMP. While one micro-CT study showed that undifferentiated BM-MSC transfected with BMP-2 and BMP-7 failed to promote increased bone formation in DO defects in a rabbit model, ${ }^{11}$ another demonstrated that forced BMP-7 expression improved the ability of BM-MSC to promote bone formation in a rat model of DO, as demonstrated by radiographic and histomorphometric analyses. ${ }^{13}$ 
Finally, bFGF transfected BM-MSC promoted greater bone mineral content and bone volume, higher bone density and increased trabecular number and thickness as compared to non-transfected BM-MCS and negative control treatment. ${ }^{14}$

The combined effect of pre-differentiated MSC and biologically active molecules

A combination of osteogenically differentiated MSC and platelet rich plasma (PRP) has been tested for the treatment of DO defects in only one study. In a rabbit model, the adjunctive use osteogenically differentiated BM-MSC significantly enhanced new bone formation and radiographic bone density in DO defects treated with PRP. ${ }^{15}$

\section{Discussion}

Tissue engineering has been proposed as an adjunct therapy to boost and overcome limitations associated with DO. Along with these lines, the use of MSC has been evaluated in several studies in an attempt to accelerate ossification and consolidation processes, and therefore, increase bone formation. ${ }^{20}$ Thus, this systematic review provides evidence on the efficacy of locally applied MSC in preclinical models of maxillary and mandibular DO. The included studies, however, exhibited an overall high risk of bias. This in turn, seriously weakens confidence in the results and may curtail potential clinical applications of stem cell-based therapies in DO.

Cell source is expected to impact the ability of MSC to efficiently differentiate in bone forming cells. ${ }^{21}$ Because of their ability to differentiate into multiple different cell types, BM-MSCs are frequently employed as a source of regenerative cells in various tissues, including bone. ${ }^{22}$ Seven trials evaluated the effect of locally applied BM-MSC on DO outcomes. ${ }^{10,13,14,15,16,17,18}$ Among those, five used undifferentiated BM-MSC. ${ }^{11,14,16}$ Four of these studies demonstrated that local application of undifferentiated BM-MSC resulted in increased bone formation in DO gap defects. ${ }^{12,14,16}$ One micro-CT study, however, concluded that undifferentiated BM-MSC failed to promote increased DO bone formation, as compared to the control treatment. ${ }^{11}$
Although the basis for this difference remains unknown, it is important to highlight that the conclusions of the last study were solely based on microtomographic data. ${ }^{11}$ This is of relevance, as the correlation between microtomographic and histomorphometry data for assessment of new bone formation has been reported as weak. ${ }^{23}$ Interestingly, a gross description of the histological findings from the above mentioned study revealed that BM-MSCtreated sites exhibited more mature medullary and cortical bones than control defects. ${ }^{11}$ Further supporting the notion that micro CT still needs to improve to differentiate woven from lamellar bone. ${ }^{24}$ Finally, the number of transplanted cells and the origin of BM-MSCs (autogenous or allogenous) used in the micro-CT study were not informed. ${ }^{11}$ Thus, it is not possible to exclude the possibility that the lower results reported in the micro-CT study might be explained by differences related to cell numbers and cell populations.

Recent evidence has highlighted that the high heterogeneity of clonally expanded MSC, characterized by differences in stages of lineage commitment, expansion capabilities and phenotypes, are expected to determine their regenerative potential and clinical efficacy. ${ }^{25}$ The studies included in this systematic review used cells from different sources, with distinct phenotypes and isolated under various protocols, all of which may impact their bone forming efficacy. Additionally, protocols also varied according to the time of the delivery and the use of scaffolds. Thus, future studies should focus on defining ideal in vitro MSC phenotypes and clinical protocols to boost DO bone formation.

Only one study compared the use of undifferentiated and osteogenically differentiated BM-MSC on DO bone formation. ${ }^{8}$ Results from this study showed that osteogenically differentiated BM-MSC arranged in cell sheets promoted greater bone formation and remodeling into mature cortical bone, than undifferentiated BM-MSC sheets and negative control treatment. ${ }^{8}$ This finding suggests the hypothesis that locally delivered of osteogenically pre-differentiated MSC may have a positive effect on promoting DO bone formation; nevertheless further investigations are needed to validate this finding. 
Only two studies employed MSC other than BM-MSC in DO defects. ${ }^{9,10}$ In one of these studies, locally administered SHED had a positive effect on DO bone formation..$^{10}$ Although SHED are multipotent and highly proliferative cells, ${ }^{26,27} \mathrm{BM}-\mathrm{MSC}$ s are still the mostly used cells for bone regeneration due to their greater potential for osteogenic differentiation. ${ }^{28}$ Therefore, despite the positive outcomes of locally delivered SHED cells in the treatment of DO defects, ${ }^{10}$ additional studies are needed to validate these preliminary results and to compare their effectiveness to the one of BM-MSC for DO bone formation. The second study failed to demonstrate a positive effect of locally delivered ADSC on DO bone formation as compared to the control group. ${ }^{9}$ Although ADSC undergo osteogenic differentiation and have been used for bone regeneration due to their wide availability and easy to obtain, ${ }^{29,30}$ these cells have been reported to have a lower osteogenic potential as compared to BM-MSC. ${ }^{31,32}$

The combined effect of locally delivered MSC and biologically active molecules has been tested in six studies. ${ }^{9,11,12,13,14,15}$ MSC gene transfection has become an exciting and promising strategy in MSC regenerative therapy. ${ }^{33}$ Along with these lines, a few DO studies used Runx $2,{ }^{9}$ OSX,12 BMP-2/7,11 BMP$2^{13}$ and $b F G F^{14}$ genetically modified MSC to favor their differentiation into osteogenic cell lineages, and further improve bone formation., ${ }^{911,12,13,14}$ Runx2 is an essential gene required for the osteoblastic differentiation and bone tissue formation, ${ }^{34,35}$ whose expression can be induced both by BMP-2 and BMP7. ${ }^{36}$ Forced expression of Runx 2 increased the ability of ADSC to promote bone formation in DO defects, ${ }^{9}$ supporting the role of Runx2 in bone formation. OSX is a zinc finger-containing transcription factor essential for osteoblast differentiation, endochondral and intramembranous bone formation. ${ }^{37,38,39,40}$ In line with its biological activity, OSX forced expression also increased the ability of BM-MSC to promote bone formation in DO defects. ${ }^{12}$

BMPs form the most extensive subgroup of the transforming growth factors- $\beta$ (TGF- $\beta$ ) superfamily of cytokines, ${ }^{41,42}$ whose main function is to promote bone formation by directing MSC differentiation into osteoblasts. ${ }^{43}$ Among all the bone morphogenetic proteins, BMP-2 and BMP-7 have been tested alone or in combination with MSC in different experimental models of bone regeneration with variable results. ${ }^{44}$ In this systematic review, two studies reported conflicting results on the efficacy of BMP to further improve the osteogenic results of undifferentiated MSC in DO defects. ${ }^{11,13}$ While one study showed that undifferentiated BM-MSC transfected with BMP-2 and BMP-7 failed to improve bone formation in DO defects in a rabbit model, ${ }_{11}^{11}$ another demonstrated that forced BMP-7 expression improved the ability of BM-MSC to promote DO bone formation in a rat model. ${ }^{13}$ These conflicting results might be explained by methodological differences between the studies, such as: the animal models, DO defect sizes, DO latency period and observation time, distraction rate and rhythm. On the last matter, it has been demonstrated that distinct distraction rates differentially regulate BMP expression in DO defect, which ultimately, have a therapeutic impact on DO outcomes. ${ }^{45}$ Lastly, these studies also differed in their method of analysis for the bone formation, with histological analysis being employed only in the study that supported the therapeutic benefits of BMP-7 gene therapy. ${ }^{13}$

Finally, one study evaluated the therapeutic efficacy of bFGF transfected BM-MSC in DO bone formation. ${ }^{14}$ bFGF is a pleiotropic growth factor that normalize cell proliferation, migration, and differentiation in various organs, including bone. ${ }^{46}$ bFGF enhances RUNX2 phosphorylation and functional activity. ${ }^{37}$ FGF-2 is expressed in osteoblast-lineage cells ${ }^{46}$ and its deficiency inhibits bone formation in animal models. ${ }^{47}$ bFGF transfected BM-MSC promoted greater DO bone formation than non-transfected BM-MCS and negative control treatment, ${ }^{14}$ further supporting its role on bone formation.

\section{Conclusion}

It is possible to conclude that while risk of bias and heterogeneity limited the strength of this systematic review, our results suggest that the therapeutic use of MSC is safe and may provide beneficial effects on DO bone formation. Moreover, it is reasonable to propose that genetic modification 
of these cells increase their ability to further induce DO bone formation. Finally, findings of this systematic review revealed that identification of ideal MSC populations for DO bone formation remains largely unknown. Future studies should be of high methodological quality, with a particular emphasis on identifying MSC phenotypes with higher potential to boost DO bone formation.

\section{Acknowledgements}

The authors have reported no conflicts of interest. This systematic review was self-funded.

\section{References}

1. Ilizarov GA. The tension-stress effect on the genesis and growth of tissues. Part I. The influence of stability of fixation and soft-tissue preservation. Clin Orthop Relat Res. 1989 Jan;(238):249-81.

2. Swennen G, Schliephake H, Dempf R, Schierle H, Malevez C. Craniofacial distraction osteogenesis: a review of the literature: Part 1: clinical studies. Int J Oral Maxillofac Surg. 2001 Apr;30(2):89-103. https://doi.org/10.1054/ijom.2000.0033

3. Ilizarov GA. The tension-stress effect on the genesis and growth of tissues: part II. The influence of the rate and frequency of distraction. Clin Orthop Relat Res. 1989 Feb;(239):263-85.

4. Ilizarov GA. The principles of the Ilizarov method. Bull Hosp Jt Dis Orthop Inst. 1988;48(1):1-11.

5. Junqueira L, Carneiro J. Tecido cartilaginoso. In: Junqueira L, Carneiro J. Histologia básica. 11th ed. Rio de Janeiro: Guanabara Koogan; 2008. p. 129-34.

6. Wang X, Wang Y, Gou W, Lu Q, Peng J, Lu S. Role of mesenchymal stem cells in bone regeneration and fracture repair: a review. Int Orthop. 2013 Dec;37(12):2491-8. https://doi.org/10.1007/s00264-013-2059-2

7. Yang ZH, Wu BL, Ye C, Jia S, Yang XJ, Hou R et al. Targeting P38 pathway regulates bony formation. Int J Med Sci. 2016;13(10):783-9. https://doi.org/10.7150/ijms.16663

8. Ma G, Zhao JL, Mao M, Chen J, Dong ZW, Liu YP. Scaffold-based delivery of bone marrow mesenchymal stem cell sheet fragments enhances new bone formation in vivo. J Oral Maxillofac Surg. 2017 Jan;75(1):92-104. https://doi.org/10.1016/i.joms.2016.08.014

9. Sun JJ, Zheng XH, Wang LY, Liu L, Jing W, Lin YF et al. New bone formation enhanced by ADSCs overexpressing hRunx2 during mandibular distraction osteogenesis in osteoporotic rabbits. J Orthop Res. 2014 May;32(5):709-20. https://doi.org/10.1002/jor.22590

10. Alkaisi A, Ismail AR, Mutum SS, Ahmad ZA, Masudi S, Abd Razak NH. Transplantation of human dental pulp stem cells: enhance bone consolidation in mandibular distraction osteogenesis. J Oral Maxillofac Surg. 2013 Oct;71(10):1758. el-13. https://doi.org/10.1016/i.joms.2013.05.016

11. Zhang WB, Zheng LW, Chua DT, Cheung LK. Treatment of irradiated mandibles with mesenchymal stem cells transfected with bone morphogenetic protein
2/7. J Oral Maxillofac Surg. 2012 Jul;70(7):1711-6. https://doi.org/10.1016/i.joms.2012.01.022

12. Lai QG, Yuan KF, Xu X, Li DR, Li GJ, Wei FL et al. Transcription factor osterix modified bone marrow mesenchymal stem cells enhance callus formation during distraction osteogenesis. Oral Surg Oral Med Oral Pathol Oral Radiol Endod. 2011 Apr;111(4):412-9. https://doi.org/10.1016/j.tripleo.2010.05.012

13. Hu J, Qi MC, Zou SJ, Li JH, Luo E. Callus formation enhanced by BMP-7 ex vivo gene therapy during distraction osteogenesis in rats. J Orthop Res. 2007 Feb;25(2):241-51. https://doi.org/10.1002/jor.20288

14. Jiang X, Zou S, Ye B, Zhu S, Liu Y, Hu J. bFGF-Modified BMMSCs enhance bone regeneration following distraction osteogenesis in rabbits. Bone. 2010 Apr;46(4):1156-61. https://doi.org/10.1016/i.bone.2009.12.017

15. Kinoshita K, Hibi H, Yamada Y, Ueda M. Promoted new bone formation in maxillary distraction osteogenesis using a tissue-engineered osteogenic material. J Craniofac Surg. 2008 Jan;19(1):80-7. https://doi.org/10.1010.1097/ scs.0b013e31815c93e316/j.bone.2009.12.017

16. Qi M, Hu J, Zou S, Zhou H, Han L. Mandibular distraction osteogenesis enhanced by bone marrow mesenchymal stem cells in rats. J Craniomaxillofac Surg. 2006 Jul;34(5):283-9. https://doi.org/10.1016/i.jcms.2006.02.002

17. Liberati A, Altman DG, Tetzlaff J, Mulrow C, Gøtzsche PC, loannidis JP et al. The PRISMA statement for reporting systematic reviews and meta-analyses of studies that evaluate healthcare interventions: explanation and elaboration. BMJ. 2009 Jul;339 jul21 1:b2700. https://doi.org/10.1136/bmi.b2700

18. Hooijmans CR, Rovers MM, Vries RB, Leenaars M, RitskesHoitinga M, Langendam MW. SYRCLE's risk of bias tool for animal studies. BMC Med Res Methodol. 2014 Mar;14(1):43. https://doi.org/10.1186/1471-2288-14-43

19. Zeng JJ, Guo P, Zhou N, Xie QT, Liao FC. Treatment of large bone defects with a novel biological transport disc in non-vascular transport distraction osteogenesis. Int J Oral Maxillofac Surg. 2016 May;45(5):670-7. https://doi.org/10.1016/j.ijom.2015.12.012 
20. Watanabe Y, Harada N, Sato K, Abe S, Yamanaka K, Matushita T. Stem cell therapy: is there a future for reconstruction of large bone defects? Injury. 2016 Jan;47 Suppl 1:S47-51. https://doi.org/10.1016/S0020-1383(16)30012-2.

21. Zomorodian E, Baghaban Eslaminejad M. Mesenchymal stem cells as a potent cell source for bone regeneration. Stem Cells Int. 2012;2012:980353. https://doi.org/10.1155/2012/980353

22. Potier E, Noailly J, Ito K. Directing bone marrow-derived stromal cell function with mechanics. J Biomech. 2010 Mar;43(5):80717. https://doi.org/10.1016/i.jbiomech.2009.11.019

23. Yeom H, Blanchard S, Kim S, Zunt S, Chu TM. Correlation between micro-computed tomography and histomorphometry for assessment of new bone formation in a calvarial experimental model. J Craniofac Surg. 2008 Mar;19(2):44652. https://doi.org/10.1097/SCS.0b013e318052fe05

24. Rühli FJ, Kuhn G, Evison R, Müller R, Schultz M. Diagnostic value of micro-CT in comparison with histology in the qualitative assessment of historical human skull bone pathologies. Am J Phys Anthropol. 2007 Aug;133(4):1099111. https://doi.org/10.1002/aipa.20611

25. Sivasubramaniyan K, Lehnen D, Ghazanfari R, Sobiesiak M, Harichandan A, Mortha E et al. Phenotypic and functional heterogeneity of human bone marrow- and amnion-derived MSC subsets. Ann N Y Acad Sci. 2012 Aug;1266(1):94-106. https://doi.org/10.1111/j.1749-6632.2012.06551.x

26. Miura M, Gronthos S, Zhao M, Lu B, Fisher LW, Robey PG et al. SHED: stem cells from human exfoliated deciduous teeth. Proc Natl Acad Sci USA. 2003 May;100(10):5807-12. https://doi.org/10.1073/pnas.0937635100

27. Jo YY, Lee HJ, Kook SY, Choung HW, Park JY, Chung JH et al. Isolation and characterization of postnatal stem cells from human dental tissues. Tissue Eng. 2007 Apr;13(4):767-73. https://doi.org/10.1089/ten.2006.0192

28. Isobe Y, Koyama N, Nakao K, Osawa K, Ikeno M, Yamanaka $S$ et al. Comparison of human mesenchymal stem cells derived from bone marrow, synovial fluid, adult dental pulp, and exfoliated deciduous tooth pulp. Int J Oral Maxillofac Surg. 2016 Jan;45(1):124-31. https://doi.org/10.1016/i.ijom.2015.06.022

29. Folgiero V, Migliano E, Tedesco M, lacovelli S, Bon $G$, Torre ML et al. Purification and characterization of adipose-derived stem cells from patients with lipoaspirate transplant. Cell Transplant. 2010;19(10):1225-35. https://doi.org/10.3727/09638910X519265

30. Xu Y, Malladi P, Wagner DR, Tataria M, Chiou M, Sylvester KG et al. Adipose-derived mesenchymal cells (AMCs): a promising future for skeletal tissue engineering. Biotechnol Genet Eng Rev. 2006;23(1):291-308. https://doi.org/10.1080/02648725.2006.10648089

31. Wu W, Le AV, Mendez JJ, Chang J, Niklason LE, Steinbacher DM. Osteogenic performance of donor-matched human adipose and bone marrow mesenchymal cells under dynamic culture. Tissue Eng Part A. 2015 May;21(9-10):1621-32. https://doi.org/10.1089/ten.tea.2014.0115
32. Ceccarelli G, Bloise N, Mantelli M, Gastaldi G, Fassina $L$, De Angelis MG et al. A comparative analysis of the in vitro effects of pulsed electromagnetic field treatment on osteogenic differentiation of two different mesenchymal cell lineages. Biores Open Access. 2013 Aug;2(4):283-94. https://doi.org/10.1089/biores.2013.0016

33. Park JS, Yi SW, Kim HJ, Kim SM, Park KH. Regulation of cell signaling factors using plga nanoparticles coated/loaded with genes and proteins for osteogenesis of human mesenchymal stem cells. ACS Appl Mater Interfaces. 2016 Nov;8(44):30387-97. https://doi.org/10.1021/acsami.6b08343

34. Franceschi RT, Xiao G. Regulation of the osteoblast-specific transcription factor, Runx2: responsiveness to multiple signal transduction pathways. J Cell Biochem. 2003 Feb;88(3):44654. https://doi.org/10.1002/jcb.10369

35. Zhang X, Yang M, Lin L, Chen P, Ma KT, Zhou CY et al. Runx2 overexpression enhances osteoblastic differentiation and mineralization in adipose: derived stem cells in vitro and in vivo. Calcif Tissue Int. 2006 Sep;79(3):169-78. https://doi.org/10.1007/s00223-006-0083-6

36. Sharff KA, Song WX, Luo X, Tang N, Luo J, Chen $J$, et al. Heyl basic helix-loop-helix protein plays an important role in mediating BMP9-induced osteogenic differentiation of mesenchymal progenitor cells. J Biol Chem. 2009 Jan;284(1):649-59. https://doi.org/10.1074/jbc.M806389200

37. Javed A, Chen H, Ghori FY. Genetic and transcriptional control of bone formation. Oral Maxillofac Surg Clin North Am. 2010 Aug;22(3):283-93. https://doi.org/10.1016/i.coms.2010.05.001

38. Zhou X, Zhang Z, Feng JQ, Dusevich VM, Sinha K, Zhang $\mathrm{H}$ et al. Multiple functions of Osterix are required for bone growth and homeostasis in postnatal mice. Proc Natl Acad Sci USA. 2010 Jul;107(29):12919-24. https://doi.org/10.1073/pnas.0912855107

39. Nakashima K, Zhou X, Kunkel G, Zhang Z, Deng JM, Behringer RR et al. The novel zinc finger-containing transcription factor osterix is required for osteoblast differentiation and bone formation. Cell. 2002 Jan;108(1):1729. https://doi.org/10.1016/S0092-8674(01)00622-5

40. Day TF, Guo X, Garrett-Beal L, Yang Y. Wnt/beta-catenin signaling in mesenchymal progenitors controls osteoblast and chondrocyte differentiation during vertebrate skeletogenesis. Dev Cell. 2005 May;8(5):739-50. https://doi.org/10.1016/i.devcel.2005.03.016

41. Beederman M, Lamplot JD, Nan G, Wang J, Liu X, Yin L et al. BMP signaling in mesenchymal stem cell differentiation and bone formation. J Biomed Sci Eng. 2013 Aug;6(08 8A):3252. https://doi.org/10.4236/jbise.2013.68A1004

42. Hinck AP. Structural studies of the TGF- $\beta$ s and their receptors: insights into evolution of the TGF- $\beta$ superfamily. FEBS Lett. 2012 Jul;586(14):1860-70. https://doi.org/10.1016/j.febslet.2012.05.028 
43. Einhorn TA, Majeska RJ, Mohaideen A, Kagel EM, Bouxsein ML, Turek TJ et al.

A single percutaneous injection of recombinant human bone morphogenetic protein-2 accelerates fracture repair. J Bone Joint Surg Am. 2003 Aug;85-A(8):1425-35. https://doi.org/10.2106/00004623-200308000-00002

44. Oreffo RO, Cooper C, Mason C,

Clements M. Mesenchymal stem cells: lineage, plasticity, and skeletal therapeutic potential. Stem Cell Rev. 2005;1(2):169-78. https://doi.org/10.1385/SCR:1:2:169
45. Cheung LK, Zheng LW, Ma L. Effect of distraction rates on expression of bone morphogenetic proteins in rabbit mandibular distraction osteogenesis. J Craniomaxillofac Surg. 2006 Jul;34(5):263-9. https://doi.org/10.1016/i.jcms.2006.02.004

46. Takei Y, Minamizaki T, Yoshiko Y. Functional diversity of fibroblast growth factors in bone formation. Int J Endocrinol. 2015;2015:729352. https://doi.org/10.1155/2015/729352PMID:25873956

47. Ornitz DM, Marie PJ. FGF signaling pathways in endochondral and intramembranous bone development and human genetic disease. Genes Dev. 2002 Jun;16(12):1446-65. https://doi.org/10.1101/gad.990702 